\title{
Características del análisis didáctico realizado por profesores para justificar la mejora en la enseñanza de las matemáticas
}

\section{Characteristics of the didactic analysis carried out by teachers to justify the improvement of mathematics teaching}

\begin{abstract}
Resumen
Presentamos las características del análisis didáctico, realizado por 25 profesores en sus trabajos de fin de máster, para justificar que sus propuestas mejoran la enseñanza de las matemáticas. Primero, se clasifican las propuestas según el tipo de innovación y las fases del proceso de instrucción. Después, se usan los criterios de idoneidad didáctica para organizar las razones que dan los autores para justificar la calidad de sus propuestas. Los resultados muestran que: a) se tienen en cuenta tres tipos de innovaciones: matemática, uso de recursos e incorporación de valores; b) las justificaciones se basan, sobre todo, en el uso implícito de criterios de idoneidad didáctica, en particular del epistémico, ecológico y mediacional y c) los profesores que implementaron sus propuestas didácticas usaron los criterios de forma más amplia y detallada que aquellos que no las implementaron.
\end{abstract}

Palabras clave: Máster Profesional en Matemáticas. Trabajo de Fin de Máster. Criterios de Idoneidad Didáctica. Enfoque Ontosemiótico. Formación de Profesores.

\begin{abstract}
The characteristics of the didactic analysis carried out by 25 teachers in their master's dissertations are presented to justify that their proposals improve mathematics teaching. First, the proposals are classified according to the type of innovation and the phases of the training process. Afterwards, the didactic suitability criteria are used to organize the reasons teachers give to justify the quality of their proposals. The results show that: a) three types of innovations are taken into account: mathematics, use of resources, and incorporation of values; b) justifications are based, above all, in the implicit use of criteria of didactic suitability, in particular epistemic, ecological and mediational, and c) the teachers who implemented their didactic proposals used the criteria in a broader and more detailed way than those who did not implement them.
\end{abstract}

Keywords: Professional Master in Mathematics. Final Master Thesis. Didactic Suitability Criteria. Ontosemiotic Approach. Teacher Training.

\footnotetext{
* Doctora en Educación en Ciencias y Matemáticas por la Pontifícia Universidade Católica do Rio Grande do Sul (PUCRS). Docente e Investigadora en la Universidad de Barcelona (UB), Barcelona, España. Dirección postal: Departamento de Educación Lingüística y Literaria y de Didáctica de las CCEE y de la Matemática, Facultad de Educación, Passeig de la Vall d’Hebrón, 171, Barcelona, España, código postal 08035. E-mail: adriana.breda@ub.edu.
} 


\section{Introducción}

Las políticas de formación continuada del profesorado tienen, por objetivo general, conseguir que los profesores realicen prácticas innovadoras, que sean cada vez mejores y de más calidad. Si bien hay diferentes políticas de formación continuada (ROESKEN, 2011), hay dos modelos claramente diferenciados. En el primero, se realizan asesoramientos en el propio centro educativo, para conseguir una reflexión crítica sobre la propia práctica, mientras que en el segundo se ofrecen cursos de formación permanente en los que el profesor se inscribe a título personal.

Con relación al segundo modelo, algunas investigaciones indican que dichos programas, para que sean eficaces, deben promover la profundización de los saberes de la disciplina sin disociarlos de los saberes pedagógicos y, además, deben desarrollar en el profesor la capacidad de reflexionar y analizar su propia práctica, de tal modo que él pueda evaluar lo que ha hecho y buscar estrategias de mejora (MOREN; DOS SANTOS, 2011; GUSKEY, 2003; GUSKEY; YOON, 2009; INGVARSON; MEIERS; BEAVIS, 2005; LEE, 2005).

En un intento de formar a los profesores de matemáticas en ejercicio se aprobó, en 2010, el Mestrado Profissional em Matemática em Rede Nacional (PROFMAT). Este máster se constituye como un curso de postgrado, ofertado en todo el territorio nacional de Brasil, y es coordinado por la Sociedade Brasileira de Matemática (SBM). Su principal objetivo es la mejora de la formación profesional, con énfasis en el dominio del contenido matemático, con la finalidad de estimular la mejora de la enseñanza de las matemáticas en todos los niveles educativos. De las orientaciones establecidas en el PROFMAT, una de ellas exige que el trabajo de fin de máster (TFM) debe ser innovador y basarse en temas específicos del currículo de matemáticas de la Educación Básica (BRASIL, 2013b).

Dadas las características del programa, descritas anteriormente, el trabajo que se presenta pretende responder a la siguiente pregunta: ¿de qué manera los profesores que cursan el PROFMAT conciben la mejora de la enseñanza de las matemáticas? Se trata de una pregunta que está relacionada con una problemática teórica más general, que podríamos formular de la siguiente manera: ¿qué papel tienen las valoraciones y los principios normativos en la práctica del profesor? De acuerdo con la primera pregunta formulada, se tiene por objetivo general investigar las características del análisis didáctico realizado por los profesores para justificar que sus propuestas didácticas son innovadoras y representan una mejora en la enseñanza de las matemáticas. 
Dicho objetivo se ha concretado en cuatro objetivos específicos que pretenden determinar: i) los tipos de propuestas de innovación desarrolladas por los profesores en sus TFM, ii) las fases del proceso de instrucción contempladas en los TFM (planificación, implementación y rediseño); iii) las características del análisis didáctico realizado por los profesores, y iv) la relación que hay entre las propuestas que fueron implementadas y el nivel de profundidad de análisis didáctico realizado por los docentes.

Asumimos la idea de innovación tal como lo proponen Century y Cassata (2016), es decir, lo entendemos como un constructo general que puede abarcar no sólo diseños didácticos para el aula, sino también programas, intervenciones, procesos, aproximaciones, métodos, estrategias, tecnologías o incluso políticas educativas.

En el caso de los TFM analizados se trata de propuestas de aula, por tanto, es un tipo muy específico de innovación. Aunque es discutible que toda innovación se pueda considerar una mejora, las orientaciones del TFM y, más en general de todo el PROFMAT, pedían a los alumnos que realizaran propuestas didácticas de aula que fuesen innovadoras y que, a su vez, representasen una mejora en relación a las propuestas que habitualmente se implementaban. En ese sentido, los profesores debían proponer una innovación y justificar didácticamente que se trataba de una mejora.

\section{Marco Teórico}

En este trabajo partimos de que el TFM implica un ejercicio de análisis didáctico, dado que se debe explicar una propuesta didáctica y justificar que ésta significa una mejora para la enseñanza de las matemáticas. Ahora bien, en el campo de la Educación Matemática no hay un consenso sobre los métodos para la valoración y mejora de los procesos de enseñanza y aprendizaje de las matemáticas. Básicamente, existen dos maneras de afrontar esta problemática, desde una perspectiva positivista o desde una consensual (FONT; GODINO, 2011).

Desde la primera, la investigación científica realizada en el área de Didáctica de las Matemáticas nos dirá cuáles son las causas que hay que modificar para conseguir los efectos considerados como objetivos a alcanzar (BARALLOBRES, 2016). Desde la perspectiva consensual, aquello que nos dice cómo guiar la mejora de los procesos de instrucción de las matemáticas, debe emanar del discurso argumentativo de la comunidad educativa (profesores, instituciones educativas, comunidad escolar, instituciones de investigación científica etc.), cuando ésta está orientada a conseguir un consenso sobre lo que se puede considerar como 
mejor. El Enfoque Ontosemiotico de la Cognición e Instrucción Matematica (EOS) (GODINO; BATANERO; FONT, 2007, 2019) se ha posicionado en esta segunda manera de entender la mejora de la enseñanza de las matemáticas, desarrollando el constructo criterios de idoneidad didáctica (CI) (BREDA; FONT; PINO-FAN, 2018) que es el principal referente teórico de esta investigación.

\subsection{Criterios de idoneidad didáctica}

Los CI deben ser entendidos como normas de corrección emanadas del discurso argumentativo de la comunidad educativa, cuando está orientada a conseguir un consenso sobre lo que se puede considerar mejor (GODINO et al., 2009). Se trata de una noción inspirada en la idea de la teoría consensual de la verdad de Peirce, y de sus desarrollos realizados por Apel (1997) y Habermas (1997); pero, que también tiene en cuenta los puntos de vista que señalan la importancia del poder en la producción y en el funcionamiento y mantenimiento de consensos (FOUCAULT, 1998). Desde esta perspectiva, la Didáctica de las Matemáticas nos puede ofrecer principios provisionales, consensuados por la comunidad interesada, que pueden servir para guiar y valorar los procesos de enseñanza y aprendizaje de las matemáticas.

Tal como se explica en Breda, Font y Pino-Fan (2018), las tendencias en la enseñanza de las matemáticas son una primera manera de observar consensos en la comunidad de la Didáctica de las Matemáticas, dado que pueden ser consideradas como regularidades que se hallan en los discursos sobre la mejora de la enseñanza de las matemáticas (GUZMÁN, 2007).

Un caso paradigmático de reconversión de algunas de estas tendencias en principios explícitos, es el caso de los principios y estándares del National Council of Teachers of Mathematics (NCTM, 2000). En el proceso seguido para establecer, con un amplio consenso, dichos principios y estándares intervinieron profesores, asociación de profesores, formadores de profesores de matemáticas, representantes de las administraciones educativas, investigadores y matemáticos, todos ellos con gran experiencia educativa.

Por otra parte, en la Didáctica de las Matemáticas (DM) se han generado conocimientos y resultados que gozan de amplio consenso (BIKNER-AHSBAHS; PREDIGER, 2010). Una característica de muchos enfoques teóricos del área es que, además de asumir unos principios para el desarrollo de su construcción teórica, consideran que estos principios deben tenerse en cuenta en la enseñanza de las matemáticas para que ésta sea 
mejor, de más calidad (BREDA; FONT; PINO-FAN, 2018).

Para el desarrollo del constructo idoneidad didáctica, se han considerado las tendencias actuales sobre la enseñanza de las matemáticas (la incorporación de nuevos contenidos, presentación de una matemática contextualizada, dar importancia a la enseñanza de los procesos matemáticos, enseñanza y aprendizaje de tipo activo, considerar que saber las matemáticas implica ser competente en su aplicación a contextos extramatemáticos, principio de equidad y la incorporación de nuevas tecnologías de la información y la comunicación), los principios del NCTM y los aportes de los diferentes enfoques teóricos del área de Didáctica de las Matemáticas (GODINO, 2013; BREDA; FONT; PINO-FAN, 2018).

En el EOS se entiende la idoneidad didáctica de un proceso de enseñanza-aprendizaje como el grado en que éste (o una parte del mismo) reúne ciertas características que permiten calificarlo como idóneo (óptimo o adecuado) para conseguir la adaptación entre los significados personales logrados por los estudiantes (aprendizaje) y los significados institucionales pretendidos o implementados (enseñanza), teniendo en cuenta las circunstancias y recursos disponibles (entorno).

Tal como se explica en Breda, Font y Pino-Fan (2018), las decisiones tomadas para delimitar las bases para el desarrollo del constructo CI fueron:

1) Debe ser una herramienta que permita al profesor reflexionar sobre su práctica y poder guiar su mejora en el contexto donde se realiza.

2) Utilizar un término que tenga un cierto aire de familia con el término calidad, pero en el que los aspectos contextuales sean más predominantes que los estructurales o inherentes. Por esta razón, se optó por el término idoneidad para introducir el constructo CI.

3) Considerar que la DM puede generar principios provisionales (un tipo de normas, llamados aquí criterios de idoneidad) consensuados por un sector importante de la comunidad interesada en la educación matemática, que pueden servir primero para guiar los procesos de enseñanza y aprendizaje de las matemáticas y, segundo, para valorar sus implementaciones.

4) El constructo $\mathrm{CI}$ debe ser multidimensional y descomponerse en idoneidades parciales (Idoneidad Epistémica, Idoneidad Cognitiva, Idoneidad Interaccional, Idoneidad Mediacional, Idoneidad Emocional e Idoneidad Ecológica), y, a su vez, cada una de ellas en componentes e indicadores (GODINO, 2013).

5) Un proceso de enseñanza y aprendizaje se considera idóneo cuando se consigue un equilibrio entre los diferentes criterios parciales de idoneidad, y no cuando sólo se dan algunos de ellos. 
6) Los criterios de idoneidad parciales (en tanto que consensos a priori) pueden entrar en conflicto con el contexto en que trabaja el profesor, lo cual conlleva, primero, tratar los CI de manera conjunta (y no como criterios independientes, como frecuentemente se hace en el caso de la calidad) y, segundo, a cuestionar o relativizar la validez de un determinado criterio en un contexto específico, lo cual lleva a dar pesos relativos diferentes a cada criterio en función del contexto.

Esta sexta decisión es posible porque los CI se consideran como normas que son principios, en lugar de normas que son reglas. Los principios tienen un aspecto de peso o importancia que las reglas no tienen, de modo que los conflictos entre principios se resuelven por peso. Dicho de otra manera, los CI, en tanto que principios, no son binarios, son graduales.

7) La posible contradicción entre la quinta y la sexta decisión se puede resolver mediante el rediseño del proceso de enseñanza y aprendizaje. En efecto, de acuerdo con la sexta decisión, el mayor peso dado a algunos principios en función del contexto inclina las decisiones en una dirección. Ahora bien, los principios con menor peso sobreviven intactos aun cuando no prevalezcan, lo cual permite darles más peso en un rediseño del proceso de enseñanza y aprendizaje de cara a una implementación futura más equilibrada.

\subsection{Componentes e indicadores de los criterios de idoneidad didáctica}

La operatividad de los criterios de idoneidad didáctica exige definir un conjunto de indicadores observables, que permitan valorar el grado de idoneidad de cada una de las facetas del proceso de instrucción. Godino et al. (2006) aportan un sistema de indicadores que sirve de guía de análisis y valoración de la idoneidad didáctica, los cuales están pensados para un proceso de instrucción en cualquier etapa educativa. Hemos considerado pertinente utilizar la adaptación de dichos componentes e indicadores publicado en Breda y Lima (2016). Por cuestiones de espacio presentamos, en el Cuadro 1, los componentes e indicadores de la idoneidad epistémica, la lista completa se puede encontrar en Breda, Pino-Fan y Font (2017) y Breda, Font y Pino-Fan (2018).

\begin{tabular}{|c|l|}
\hline \multicolumn{1}{|c|}{ Indicadores } \\
\hline Componentes & \multicolumn{1}{|c|}{ Idoneidad Epistémica } \\
\hline Errores & $\begin{array}{l}\checkmark \text { No se observan prácticas que se consideren incorrectas } \\
\text { desde el punto de vista matemático. }\end{array}$ \\
\hline Ambigüedades & $\begin{array}{l}\checkmark \text { No se observan ambigüedades que puedan llevar a la } \\
\text { confusión a los alumnos: definiciones y procedimientos clara y } \\
\text { correctamente enunciados, adaptados al nivel educativo al que se } \\
\text { dirigen; adecuación de las explicaciones, comprobaciones, }\end{array}$ \\
\hline
\end{tabular}




\begin{tabular}{|c|c|}
\hline & $\begin{array}{l}\text { demostraciones al nivel educativo a que se dirigen, uso controlado } \\
\text { de metáforas, etc. }\end{array}$ \\
\hline Riqueza de procesos & $\begin{array}{l}\checkmark \quad \text { La secuencia de tareas contempla la realización de } \\
\text { procesos relevantes en la actividad matemática (modelización, } \\
\text { argumentación, resolución de problemas, conexiones etc.). }\end{array}$ \\
\hline $\begin{array}{l}\text { Representatividad de la } \\
\text { complejidad }\end{array}$ & $\begin{array}{l}\checkmark \quad \text { Los significados parciales (definiciones, propiedades, } \\
\text { procedimientos etc.) son una muestra representativa de la } \\
\text { complejidad contemplada en el currículo de la noción matemática } \\
\text { que se quiere enseñar. } \\
\checkmark \quad \text { Los significados parciales (definiciones, propiedades, } \\
\text { procedimientos etc.) son una muestra representativa de la } \\
\text { complejidad de la noción matemática que se quiere enseñar. } \\
\checkmark \quad \text { Para uno o varios significados parciales seleccionados, } \\
\text { muestra representativa de problemas. } \\
\checkmark \quad \text { Para uno o varios significados parciales seleccionados, uso } \\
\text { de diferentes modos de expresión (verbal, gráfico, simbólico...), } \\
\text { tratamientos y conversiones entre los mismos. }\end{array}$ \\
\hline
\end{tabular}

Cuadro 1 - Componentes e indicadores de la Idoneidad Epistémica

Fuente: Breda, Pino-Fan y Font (2017).

Tal como se ha mencionado, tanto los componentes como los indicadores de los CI se han confeccionado teniendo en cuenta las tendencias, los principios y los resultados de la investigación en el área de Didáctica de las Matemáticas, en particular, para la idoneidad epistémica se ha tenido en cuenta el principio: los objetos matemáticos emergen de las prácticas, lo cual conlleva su complejidad (RONDERO; FONT, 2015). De este principio se deriva un componente (representatividad de la complejidad) cuyo objetivo es que se tenga en cuenta la complejidad matemática en el diseño y rediseño de las secuencias didácticas.

\section{Metodología}

En este apartado se explican algunas de las características del programa PROFMAT y de los profesores participantes. Se detalla el proceso de recolección y análisis de los datos y se muestra un ejemplo concreto de cómo se examinaron, usando los criterios de idoneidad didáctica, los análisis didácticos realizados por los profesores en sus TFM.

\subsection{Características del programa y de los profesores participantes}

Una de las principales características del PROFMAT es la importancia que atribuye a la formación de los profesores en el contenido matemático. El candidato ingresa en el programa después de superar una prueba de matemática compuesta por 38 cuestiones relacionadas con: conjuntos numéricos, geometría, magnitudes y medidas, resolución de problemas, álgebra y tratamiento estadístico de la información. A lo largo de dos años 
completos, el estudiante tiene que cursar asignaturas obligatorias de matemáticas superiores (funciones, matemática discreta, aritmética, álgebra, geometría, cálculo, resolución de problemas), disciplinas electivas (recursos computacionales, historia de las matemáticas, otras asignaturas de matemáticas) y el trabajo de fin de máster (TFM).

Desde el punto de vista de la Didáctica de la Matemática, el programa ofrece dos asignaturas opcionales (no obligatorias): Matemática y Actualidad y Modelamiento Matemático. Para obtener el título, además de aprobar las asignaturas, el estudiante debe superar un examen de calificación (prueba de matemáticas) y debe defender con éxito el TFM. Otro aspecto que resaltar es que, según el reglamento del programa, un $80 \%$ de las plazas están destinadas a profesores que imparten clases de matemáticas en las escuelas públicas de la Educacion Básica (Fundamental I, II y Enseñanza Media).

\section{Características del TFM}

El TFM, además de ser innovador, debe desarrollar temas específicos del currículo de matemáticas de la Educación Básica, materializándose en un proyecto basado en una propuesta didáctica con aplicación directa al aula de matemáticas. La asignatura del TFM se desarrolla en un tiempo de dos meses (enero y febrero del último año) con la recomendación de que el trabajo sea iniciado, por lo menos, en el inicio del semestre anterior al previsto para la conclusión del TFM. La elaboración del trabajo final de máster es acompañada por un proceso de tutorización (a distancia), dónde el tutor tiene formación, mayoritariamente, en matemática pura.

\subsection{Selección de los datos y etapas del análisis}

Se ha utilizado una metodología de investigación cualitativa que se basa en la comprensión e interpretación de los datos. Se seleccionaron veinticinco TFM publicados en 2013 y 2014 en el estado de Rio Gran del Sur (RS). Este estado fue seleccionado por tres razones: a) dicho estado participa del programa con dos universidades; b) presenta un número de producciones finales razonable, de manera que se pueden inferir conclusiones y c) la facilidad de acceso a los documentos seleccionados para el análisis. Los veinticinco TFM seleccionados fueron realizados por profesores de matemáticas en ejercicio, vinculados a escuelas públicas y privadas ubicadas en el estado de RS.

De acuerdo con los objetivos previstos, el análisis de los datos se realizó para determinar: i) los tipos de propuestas de innovación desarrollada en cada TFM, ii) las fases del proceso de instrucción contempladas (planificación, implementación y rediseño); iii) las 
características del análisis didáctico realizado para justificar que la propuesta presentada en el TFM representa una mejora de la enseñanza de las matemáticas, y iv) la relación que hay entre las propuestas que fueron implementadas y el nivel de análisis didáctico realizado.

Para clasificar los TFM con relación a los ítems (i) y (ii), se utilizó una metodología de tipo inductiva, sin categorización previa. A partir de las descripciones realizadas por los profesores en sus TFM, se pudo determinar: a) el tipo de innovación de la propuesta desarrollada en los TFM y cuáles eran los principales argumentos dados por los profesores para justificar que dicha propuesta era innovadora; b) si el profesor había realizado solamente el diseño de la propuesta o si también la había implementado y/o rediseñado.

El análisis cualitativo referente a los objetivos (iii) y (iv) se realizó usando los componentes e indicadores de los CI expuestos en Breda, Pino-Fan y Font (2017) y Breda, Font y Pino-Fan (2018). Para analizar el uso implícito de los CI en cada TFM, se estableció un nivel de escala discreta, variando de 0 a 3, donde el resultado final de dicho valor se asignó mediante una triangulación con dos especialistas del EOS. Es decir, la asignación fue realizada mediante la implicación de investigadores del mismo campo teórico a fin de tener diferentes puntos de vista durante el análisis de los TFM - es lo que Lincoln y Guba (1985) denominan Member Checking. En el Cuadro 2 se explica las características atribuidas para cada nivel.

\begin{tabular}{|c|l|}
\hline $\begin{array}{c}\text { Nivel de asignación } \\
\text { para cada CI }\end{array}$ & \multicolumn{1}{c|}{ Características } \\
\hline 0 & $\begin{array}{l}\text { En el TFM no se contempla ningún párrafo que pueda ser considerado } \\
\text { cómo evidencia del uso implícito o explícito de algún componente o } \\
\text { indicador del criterio de idoneidad didáctica que se está analizando. }\end{array}$ \\
\hline 1 & $\begin{array}{l}\text { En el TFM se presentan, de manera esporádica, algunos párafos que } \\
\text { puedan ser considerados cómo evidencia del uso implícito o explícito } \\
\text { de algún componente o indicador del criterio de idoneidad didáctica } \\
\text { que se está analizando. }\end{array}$ \\
\hline 2 & $\begin{array}{l}\text { En el TFM se contemplan algunas evidencias del uso implícito y } \\
\text { explícito de la mayoría de los componentes y sus respectivos } \\
\text { indicadores del criterio de idoneidad didáctica que se está analizando. }\end{array}$ \\
\hline 3 & $\begin{array}{l}\text { Cuando las evidencias del nivel 2 son párrafos que presentan bastante } \\
\text { detalle, profundidad y coherencia. }\end{array}$ \\
\hline
\end{tabular}

Cuadro 2 - Nivel de asignación para el uso de cada CI

Fuente: el autor

Es importante señalar que el nivel de cada criterio fue establecido, no sólo teniendo en cuenta el número de componentes e indicadores de cada criterio usados por el autor del TFM, sino también, por el detalle y la coherencia de las evidencias de dicho uso (por ejemplo, que los comentarios de los profesores no sean superficiales o intrancendentes). 


\title{
3.3 Análisis didáctico realizado por un profesor
}

En este apartado presentamos, como ejemplo, el análisis de un caso cuya propuesta innovadora consiste en el establecimiento de conexiones extramatemáticas. En particular, el TFM de Abeeg (2014), trata de implementar el estudio de las funciones lineales por medio de la modelación de situaciones contextualizadas con alumnos del octavo año de la Enseñanza Fundamental. Por cuestiones de espacio, ilustramos solo como se realizó el análisis desde el criterio epistémico.

\subsubsection{Idoneidad epistémica}

\section{Errores y ambigüedades y Riqueza de procesos}

El autor del TFM no comenta, explícitamente, que él haya cometido errores en su explicación, pero sí es consciente de que su explicación, por el tipo de notación simbólica usada para representar funciones, es ambigua y puede generar confusiones en los alumnos

\begin{abstract}
La comprensión de la función, con la terminología del concepto (conjuntos, elementos, variables dependientes e independientes) y la correspondiente simbología $\mathrm{x}, \mathrm{y}, \mathrm{f}(\mathrm{x})$, con sus diferentes representaciones, resultado de la transformación histórica, es de difícil comprensión para los profesores, estas dificultades se reflejan en el proceso de enseñanza y aprendizaje de los alumnos (ABEEG, 2014, p. 16).
\end{abstract}

Por otra parte, justifica la calidad de su propuesta innovadora argumentando que ésta permite a los alumnos realizar procesos matemáticos relacionados con el proceso de modelización matemática, además, en su relato se observa que algunos de estos procesos efectivamente se trabajaron durante la implementación de la propuesta

[...] los alumnos hacen experimentos u observaciones que permiten recoger datos, siendo que a partir de esos datos es natural que aparezca una forma de representación, a menudo una tabla. En este punto, los alumnos son llevados a explorar determinadas situaciones, donde formulan hipótesis y hacen estimaciones, de manera que la formulación del modelo matemático es consecuencia de este proceso $[\ldots]$ (ABEGG, 2014, p. 32-33).

\section{Representatividad}

El autor del TFM presenta diversos comentarios sobre la complejidad de la proporcionalidad, de las funciones y de la pendiente de la recta. Con relación a la proporcionalidad, el autor propone priorizar el enfoque funcional en su enseñanza, para ello tiene en cuenta otros enfoques y los relaciona (o no) con su propuesta. Es decir, el autor, implícitamente, primero tiene en cuenta el siguiente indicador: los significados parciales son 
una muestra representativa de la complejidad de la noción matemática que se quiere enseñar contemplada en el currículo, ya que explica que el currículo de la Enseñanza Fundamental contempla diferentes maneras de entender la proporcionalidad (aritmético-algebraico, funcional y geométrico). En este sentido, el autor afirma que su propuesta de incorporar una visión funcional a la enseñanza de la proporcionalidad en el octavo año de la Enseñanza Fundamental permite una enseñanza más completa, integrada y conectada de este objeto matemático.

En nuestra propuesta de actividad didáctica, el objeto de estudio, funciones lineales, fue escogido para dar continuidad al contenido de proporcionalidad, que muchas veces se limita sólo a la mecanización del procedimiento de la "regla de tres". Buscamos entonces una manera de dar significado al contenido de proporcionalidad, introduciendo el concepto de función lineal por medio de la modelación matemática, contextualizando una situación de la realidad (ABEGG, 2014, p. 33).

En estas reflexiones, el autor quiere priorizar el punto de vista funcional, ya que éste completa, pero no elimina los otros significados parciales de la proporcionalidad contemplados en el currículo. Además, de los tres significados comentados anteriormente, considera el significado parcial correspondiente a la teoría de las proporciones presentes en la geometría griega, que, según él, se relacionan con el significado parcial aritmético-algebraico. En cierta forma, el autor del TFM contempla cuatro de los significados parciales para la proporcionalidad considerados en Rivas (2013): matemática griega, aritmético-algebraico, funcional y geométrico.

La enseñanza de proporcionalidad directa como función lineal fue defendida por Ávila (1986), quien afirma que el abordaje escolar del tema proporcionalidad como igualdad de razones no es tan común, pues guarda restos de la teoría de las proporciones de Eudoxo, que dio lugar con el desarrollo de la Matemática, a la teoría de los números reales de Dedekind [...] (ÁVILA, 1986, p. 2 apud ABEGG, 2014, p. 21).

El objeto matemático función es un objeto que presenta gran complejidad, como muestra su evolución histórica. Font (2011) considera que esta evolución puede ser organizada en cuatro significados parciales: tabular, gráfico, analítico y conjuntista. El autor del TFM es consciente de la complejidad asociada a la noción de función, optando por presentar la función, sobre todo, como una relación entre magnitudes y tiene en cuenta que, para uno o varios significados parciales, se hace uso de diferentes modos de expresión (verbal, gráfico, simbólico, etc.), tratamientos y conversiones entre los mismos. Por otro lado, el autor opta por presentar una configuración epistémica de tipo empirista (realista, intuitiva etc.), donde la configuración conjuntista no se considera (FONT, 2011).

La noción de pendiente de la recta también tiene cierta complejidad. Azcárate (1990), considera cuatros significados parciales: a) geométrico (inclinación de la recta); b) algebraico 
(el coeficiente $a$ en la fórmula $y=a x+b)$; c) funcional (cociente entre las variables): d) trigonométrico (tangente del ángulo). El autor del TFM, por una parte, tiene en cuenta la complejidad de la noción de pendiente cuando revisa cómo los libros didácticos presentan la función lineal ya que realiza comentarios que, de manera implícita, evidencian los diferentes significados parciales de la noción de pendiente de la recta y la necesidad de su conexión. Sin embargo, por otra parte, en su propuesta de secuencia didáctica enfatiza, casi exclusivamente, la interpretación de la pendiente como tasa media de variación (significado funcional).

\subsubsection{Consideraciones sobre la asignación de valores a cada criterio de idoneidad}

El TFM termina con una reflexión final en la que el autor, de manera implícita, usa los seis criterios de idoneidad didáctica en sus valoraciones. Además, sus reflexiones y valoraciones no son superficiales. De los argumentos del autor del TFM se infiere, también, que, en cierta manera, consiguió un equilibrio en el uso de los seis CI. Con base en el análisis que se acaba de mostrar parcialmente en esta sección, la valoración del uso de cada uno de los seis criterios de idoneidad fue tres.

Por ejemplo, con relación al criterio de idoneidad epistémica el autor tiene en cuenta que: a) se podrían generar ambigüedades referente al tema propuesto, b) se crearon condiciones para que los alumnos aprendieran a recoger datos por medio de la experimentación, formular modelos matemáticos, resolverlos y validarlos, c) se buscó una cierta representatividad de la complejidad de los objetos que se querían enseñar (proporcionalidad, función y pendiente); por esas razones se asignó la nota 3 para la idoneidad epistémica.

\section{Resultados y discusión}

En esta sección se presentan los criterios usados por los profesores (y se muestran algunas evidencias que permiten inferirlos) que cursan el PROFMAT cuando justifican que sus propuestas son innovadoras y representan una mejora en la enseñanza de las matemáticas. Además, se presenta el nivel de asignación dado para el uso de cada criterio en los diferentes tipos de innovación y procesos de instrucción propuestos por los profesores. 


\subsection{Tipos de innovación y fases del proceso de instrucción}

Los resultados muestran que los profesores tienen en cuenta, básicamente, tres tipos de innovación: i) matemática, en la que se contempla la incorporación de contenidos de nivel superior en la Educación Básica, el establecimiento de conexiones intramatemáticas y extramatemáticas; ii) en recursos, que se caracteriza por la incorporación de materiales visuales y manipulativos y la incorporación de recursos informáticos; iii) en valores, dónde se introduce la idea del pensamiento crítico y la ciudadanía. A continuación, siguen unos breves párrafos tomados de tres TFM donde se ilustran, respectivamente, tres tipos de innovaciones: conexiones extramatemáticas (innovación matemática), materiales visuales y manipulativos (innovación en recursos) y pensamiento crítico y ciudadanía (innovación en valores).

Cabe resaltar que nuestra intención de presentar el contenido de funciones lineales en el $8^{\circ}$ año, no es trabajar con un lenguaje excesivamente formal, sino presentar a los alumnos situaciones contextualizadas donde se pueda explorar la dependencia entre dos magnitudes [...] (ABEGG, 2014, p. 19-20).

[...] este trabajo es innovador pues el uso de tecnología digital, específicamente softwares de geometría dinámica llevan al alumno a vincular lo que él ya sabe con el nuevo conocimiento a adquirir (...) (BASTOS, 2014, p. 17).

Esta propuesta pedagógica presentada en esta tesis, tiene como objetivo proporcionar herramientas y recursos para que los estudiantes aprendan a actuar en el mundo en el que operan, lo que lleva a identificar, interpretar, evaluar y criticar las matemáticas, para que la formación contribuya para formar ciudadanos libres y críticos, responsables de sus acciones (REIS, 2013, p. 8).

Con relación a las fases del proceso de instrucción contempladas, trece de los TFM presentan solamente la planificación, once realizan la implementación y sólo un incluye el rediseño (Cuadro 3).

\begin{tabular}{|c|c|c|c|c|}
\hline \multirow{2}{*}{\multicolumn{2}{|c|}{ Innovación }} & \multicolumn{3}{|c|}{ Proceso de Instrucción } \\
\hline & & \multirow{2}{*}{$\begin{array}{c}\text { Diseño } \\
2 \\
\end{array}$} & \multirow{2}{*}{$\begin{array}{c}\text { Implementación } \\
7 \\
\end{array}$} & \multirow{2}{*}{$\frac{\text { Rediseño }}{0}$} \\
\hline \multirow{3}{*}{ Matemática } & Contenidos de nivel superior en la Educación Básica & & & \\
\hline & Conexiones intramatemáticas & 2 & 1 & 0 \\
\hline & Conexiones extramatemáticas & 3 & 3 & 0 \\
\hline \multirow{2}{*}{ Recursos } & Informáticos & 4 & 0 & 1 \\
\hline & Materiales visuales y manipulativos & 1 & 0 & 0 \\
\hline Valores & Pensamiento crítico y ciudadanía & 1 & 0 & 0 \\
\hline
\end{tabular}

Cuadro 3 - Número de TFM con relación al tipo de innovación y fases del proceso de instrucción Fuente: el autor

Las justificaciones dadas por los profesores con relación a los tres tipos de innovación son: a) nuevas formas de relacionar y acercar los contenidos matemáticos; b) realización de procesos relevantes (por ejemplo, la generalización, la modelación, la significación); c) permiten un aprendizaje constructivista y significativo; d) permiten clases más atractivas que 
despiertan el interés de los estudiantes; e) estimula a los estudiantes a interpretar y tomar decisiones en el mundo donde viven.

\subsection{Nivel de asignación de uso de cada criterio de idoneidad en cada TFM}

Con relación a los objetivos (iii) y (iv), se evaluó el nivel del uso de cada CI (epistémico, cognitivo, mediacional, interaccional, emocional y ecológico) en cada TFM. Para ello, se utilizó la escala presentada en el Cuadro 2. En las Figuras 1, 2 y 3 se muestra el nivel de uso de los criterios presentados por los veinticinco TFM.
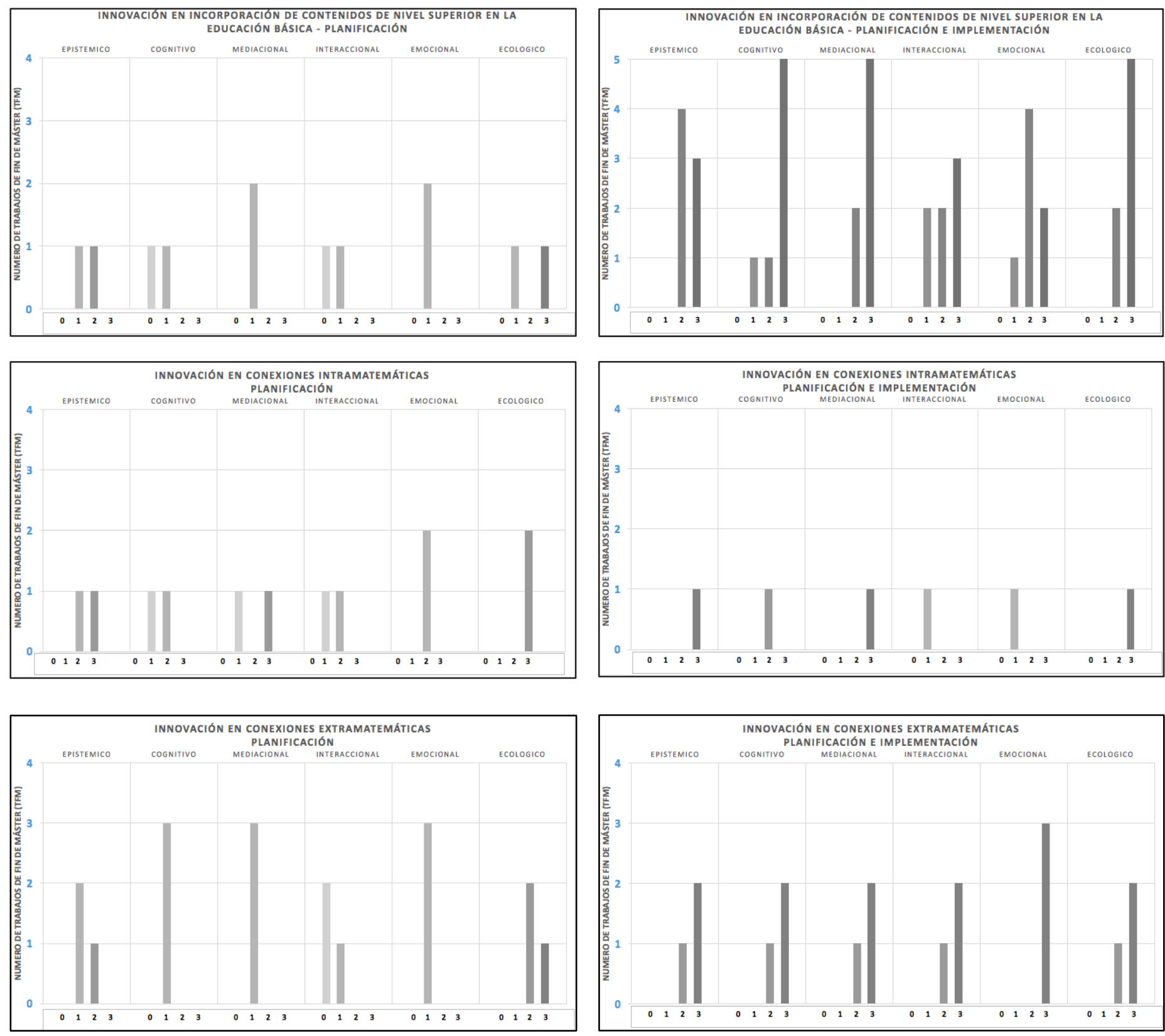

Figura 1 - Nivel de uso de los CI para los TFM que tienen innovación matemática.

Fuente: autoría propia 

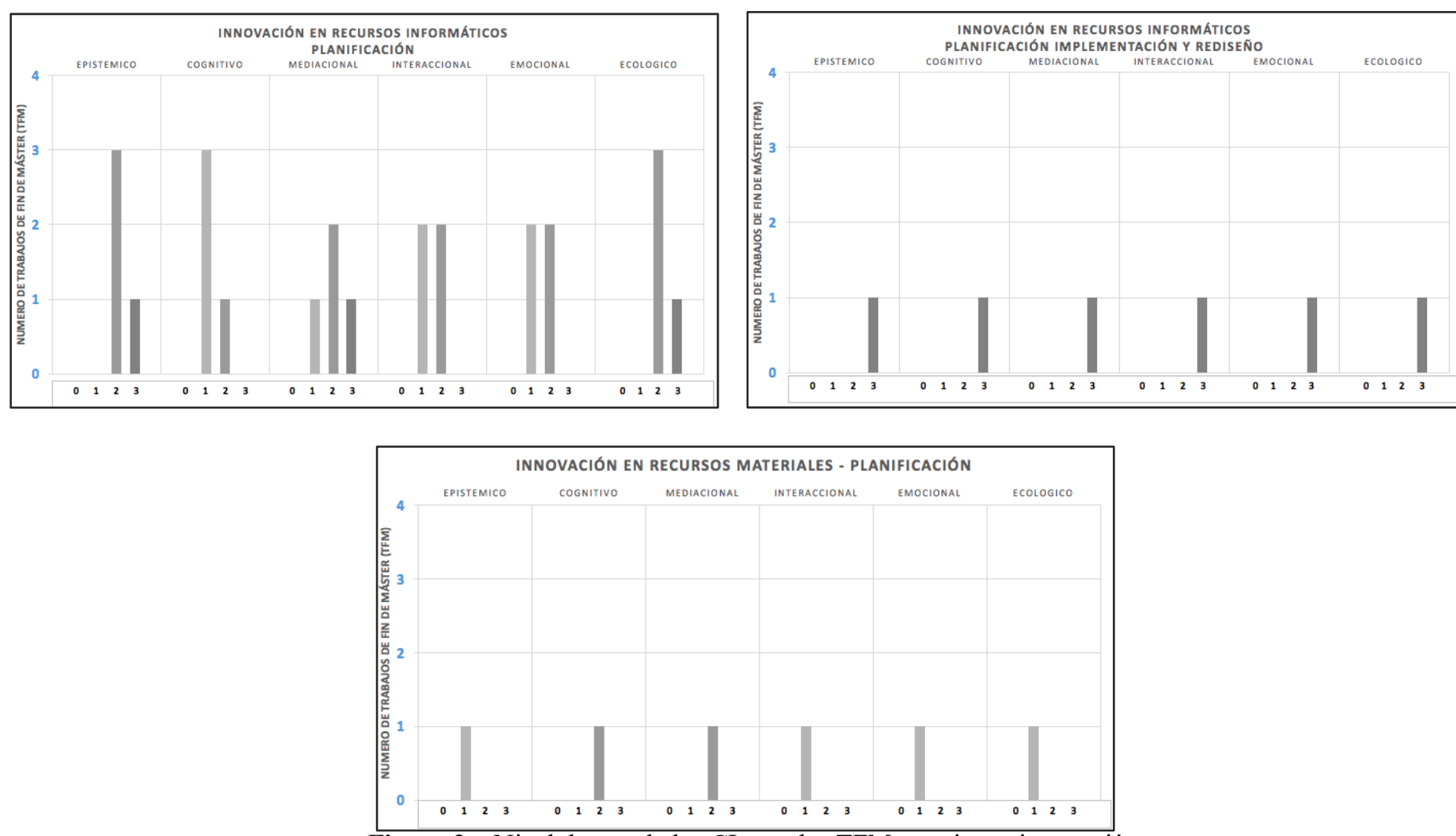

Figura 2 - Nivel de uso de los CI para los TFM que tienen innovación en recursos Fuente: autoría propia.

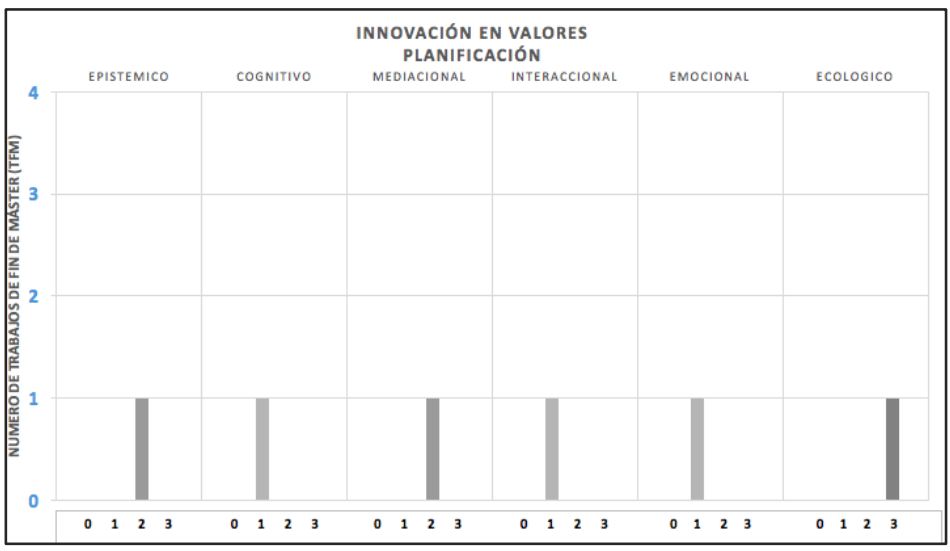

Figura 3 - Nivel de uso de los CI para los TFM que tienen innovación en valores Fuente: autoría propia

En las figuras 1, 2 y 3 se observa que los TFM que contemplan solamente el diseño de un proceso de instrucción, presentan un análisis didáctico mucho más enfocado en los aspectos epistémicos y ecológicos. Por otro lado, los TFM cuya propuesta se implementó presentan un nivel de análisis didáctico más profundo, usan más CI y consideran un cierto equilibrio entre ellos.

Tomemos, por ejemplo, las gráficas que muestran el nivel de uso de los criterios para los TFM que tienen como innovación las conexiones extramatemáticas. En relación al criterio emocional, los tres TFM que implementan la propuesta presentan el nivel 3. Por otro lado, los 
tres TFM que presentan solamente el diseño, se calificaron en el nivel 1. De manera general, se observa que las justificaciones utilizadas para argumentar que las propuestas promueven una mejora en la enseñanza de las matemáticas, se relacionan, sobre todo, con los criterios de idoneidad epistémico, ecológico y mediacional, y que otros criterios como el cognitivo, el emocional y el interaccional están poco presentes; lo mismo se puede concluir para los demás tipos de innovaciones: Contenidos de nivel superior en la Educación Básica, Conexiones Intramatemáticas y Recursos informáticos.

El TFM que basa su innovación en los Recursos Materiales y fue implementado presenta un nivel de uso del criterio cognitivo y mediacional un poco más elevado que los otros criterios y el TFM que basa su innovación en Valores, presenta un nivel de la idoneidad ecológica un poco más elevado, dado que la naturaleza de la propuesta está enfocada en valorar la utilidad laboral y social de la propuesta.

Con relación al objetivo (iv) - la relación que hay entre las propuestas que fueron implementadas y el nivel de análisis didáctico realizado - se infiere que los TFM que implementaron la propuesta didáctica presentan un uso más equilibrado y más profundo de los criterios de idoneidad didáctica, comparado con los TFM cuyas propuestas no fueron implementadas. Es decir, el nivel de análisis didáctico en los trabajos que implementan la propuesta está mucho más desarrollado y es más detallado que el que se realiza en los TFM que no contemplan la fase de implementación.

\section{Discusión y consideraciones finales}

$\mathrm{Al}$ investigar las características del análisis didáctico, realizado por los profesores para justificar que sus propuestas didácticas son innovadoras y representan una mejora en la enseñanza de las matemáticas, se encontró que la mayor parte de los trabajos proponen o bien una innovación matemática o bien una innovación en recursos informáticos. Este resultado parece coherente con el hecho de que el PROFMAT está organizado por la SBM con un programa de estudios que pone el foco, sobre todo, en aspectos matemáticos y en la incorporación de las TIC. Además, se constató que, aproximadamente, la mitad de los trabajos no han implementado la propuesta, lo cual es posible con las orientaciones del master (la no obligatoriedad de la implementación).

Con relación a los objetivos tres y cuatro, el análisis de los TFM muestra que las reflexiones de los profesores, cuando éstas son claramente valorativas, se organizan, implícitamente, usando algunos indicadores de los componentes de los criterios de idoneidad 
didáctica propuestos por el EOS. En el caso del objetivo tres, se observa que las evidencias utilizadas para justificar que las propuestas promueven una mejora en la enseñanza de las matemáticas, se relacionan, sobre todo, con los criterios de idoneidad epistémica, ecológica y mediacional y que otros criterios como el cognitivo, el emocional y el interaccional están poco presentes. Estos últimos tres criterios tienen poca presencia, sobre todo, en los TFM que no implementan la propuesta didáctica.

Respecto al objetivo cuatro (la relación que hay entre las propuestas que fueron implementadas y el nivel de análisis didáctico realizado) se concluye que los profesores que implementaron la propuesta manejan una mayor cantidad de criterios (y de sus componentes e indicadores), y, además, están preocupados por el equilibrio entre de los diferentes CI. En cambio, en el grupo de profesores que no han implementado su propuesta, no se hallan reflexiones sobre el tema del equilibrio entre criterios, lo cual es coherente con el hecho de que no han realizado la implementación, que es donde se manifiesta el problema del equilibrio.

Una limitación importante de esta investigación es su extensión, ya que los resultados están acotados a un master profesional especifico (PROFMAT), impartido en una región específica de Brasil (Rio Grande do Sul). Ahora bien, resultados similares han sido encontrados en otras investigaciones realizadas con metodologías diferentes, en contextos institucionales diferentes y en países diferentes (RAMOS, 2006; SECKEL, 2016; MORALES-LÓPEZ, 2017; MORALES-LÓPEZ; FONT, 2019; GIACOMONE; GODINO; BELTRÁN-PELLICER, 2018; ESQUÉ; BREDA, 2021).

Un aspecto, difícil de explicar, es la razón por la cual los CI funcionan implícitamente como regularidades en el discurso de los profesores, sin habérsele enseñado el uso de esta herramienta para pautar su reflexión. Una posible explicación es que la formación recibida en el PROFMAT los ha llevado a realizar este tipo de análisis. Sin embargo, la investigación de Caldatto, Pavanello y Fiorentini (2016) nos lleva a creer que las características de la formación recibida en el PROFMAT no promueven en los profesores participantes este tipo de reflexión.

Por tanto, una explicación plausible de que los criterios, sus componentes e indicadores funcionen como regularidades en el discurso del profesor es que reflejan consensos sobre cómo debe ser una buena enseñanza de las matemáticas ampliamente asumidos en la comunidad de educadores matemáticos; y es plausible pensar que el uso implícito que hace el profesor de los criterios de idoneidad didáctica se debe a su formación y experiencia previa, la cual le hace partícipe de dichos consensos. 


\section{Agradecimientos}

Este trabajo se ha realizado en el marco del contracto FJCI-2017-34021 del Programa Juan de la Cierva-Formación 2017.

\section{Referencias}

ABEEG, D. R. Função Linear por meio da Modelagem Matemática: um relato de caso nas séries finais do Ensino Fundamental. 2014. Dissertação (Mestrado Profissional em Matemática em Rede Nacional - PROFMAT) - Centro de Ciências Exatas, Universidade Federal de Santa Maria, Santa Maria, 2014.

APEL, K. O. ¿Husserl, Tarski o Peirce? Por una teoría semiótico-trascendental de la verdad como consenso. In: NICOLÁS, J. A.; FRÁPOLI, M. J. (Ed.). Teorías de la verdad en el siglo XX. Madrid: Tecnos, 1997, p. 597-616.

AZCÁRATE, C. La velocidad: introducción al concepto de derivada. 1990. Tesis (Doctorado en Didáctica de las Matemáticas) - Departamento de Didáctica de la Matemática, Universitat Autònoma de Barcelona, Barcelona, 1990.

BARALLOBRES, G. Diferentes interpretaciones de las dificultades de aprendizaje en matemática. Educación matemática, Ciudad de México, v. 28, n. 1, p. 39-68, 2016.

BASTOS, D. O. Estudo da Circunferência no Ensino Médio: Sugestões de Atividades com a Utilização do Software GeoGebra. 2014. Dissertação (Mestrado Profissional em Matemática em Rede Nacional - PROFMAT) - Instituto de Matemática, Estatística e Física, Universidade Federal do Rio Grande, Rio Grande.

BIKNER-AHSBAHS, A.; PREDIGER, S. Networking of theories - an approach for exploiting the diversity of theoretical approaches. In: SRIRAMAN, B.; ENGLISH, L. (Ed.). Theories of mathematics education: Seeking new frontiers. New York: Springer, 2010, p. 483-506.

BRASIL. Avaliação suplementar externa do programa de mestrado profissional em matemática em rede nacional (PROFMAT), CAPES, 2013b. Disponible en: http://www.profmatsbm.org.br/files/Arquivos\%20do\%20Site/Relatorio/PROFMAT_Av_Suplementar.pdf. Acceso en: día nov. 2014.

BREDA, A.; FONT, V.; PINO-FAN, L. R. Criterios valorativos y normativos en la didáctica de las matemáticas: el caso del constructo idoneidad didáctica. Bolema-Boletim de Educação Matemática, Rio Claro, v. 32, n. 60, p. 255-278, 2018.

BREDA, A.; LIMA, V. M. R. Estudio de caso sobre el análisis didáctico realizado en un trabajo final de un máster para profesores de matemáticas en servicio. REDIMAT - Journal of Research in Mathematics Education, Barcelona, v. 5, n. 1, p. 74-103, 2016.

BREDA, A.; PINO-FAN, L.; FONT, V. Meta didactic-mathematical knowledge of teachers: criteria for the reflection and assessment on teaching practice. EURASIA Journal of Mathematics, Science and Technology Education, London, v. 13, n. 6, p. 1893-1918, 2017.

CALDATTO, M. E.; PAVANELLO, R. M.; FIORENTINI, D. O PROFMAT e a Formação do Professor de Matemática: uma análise curricular a partir de uma perspectiva processual e descentralizadora. Bolema-Boletim de Educação Matemática, Rio Claro, v. 30, n. 56, p. 906-925, 
2016.

CENTURY, J.; CASSATA, A. Implementation research: Finding common ground on what, how, why, where, and who. Review of Research in Education, New York, v. 40, n. 1, p. 169-215, 2016.

ESQUÉ, D.; BREDA, A. Valoración y rediseño de una unidad sobre proporcionalidad utilizando la herramienta Idoneidad Didáctica. Uniciencia, Costa Rica, n. 35, 2021.

FONT, V. Funciones. In: GOÑI, J. M. (Ed.). MATEMÁTICAS: Complementos de formación disciplinar. Barcelona: Editorial Graó/Ministerio de Educación, 2011, p. 145-186.

FONT, V.; GODINO, J. D. Inicio a la investigación en la enseñanza de las matemáticas en secundaria y bachillerato. In: Goñi, j. M (Ed.). Matemáticas: Investigación, innovación y buenas prácticas. Barcelona, España: Graó, 2011, p. 09-55.

FOUCAULT, M. Microfísica do poder. 7. ed. Rio de Janeiro: edições Graal, 1998.

GIACOMONE, B.; GODINO, J. D.; BELTRÁN-PELLICER, P. Developing the prospective mathematics teachers' didactical suitability analysis competence. Educação e Pesquisa, São Paulo, v. 44, e172011, p. 1-21, 2018.

GODINO, J. D. Indicadores de la idoneidad didáctica de procesos de enseñanza y aprendizaje de las matemáticas. Cuadernos de Investigación y Formación en Educación Matemática, Costa Rica, v. 8, n. 11, p. 111-132, 2013.

GODINO, J. D.; BATANERO, C.; FONT, V. The onto-semiotic approach to research in mathematics education. ZDM, Berlín, v. 39, n. 1-2, p. 127-135, 2007.

GODINO, J. D.; BATANERO, C. y FONT, V. The onto-semiotic approach: implications for the prescriptive character of didactics. For the Learning of Mathematics, Edmonton, v. 39, n.1, p. 38-43, 2019.

GODINO, J. D.; BENCOMO, D.; FONT, V. y WILHELMI, M. R. Análisis y valoración de la idoneidad didáctica de procesos de estudio de las matemáticas. Paradigma, Maracay, v. 27, n. 2, p. 221-252, 2006.

GODINO, J. D.; FONT, V.; WILHELMI, M. R.; CASTRO, C. Aproximación a la dimensión normativa en Didáctica de las Matemáticas desde un enfoque ontosemiótico. Enseñanza de las Ciencias, Barcelona, v. 27, n. 1, p. 59-76, 2009.

GUSKEY, T. R. What makes professional development effective? Phi delta kappan, New York, v. 84, n. 10, p. 748-50, 2003.

GUSKEY, T. R.; YOON, K. S. What works in professional development? Phi delta kappan, New York, v. 90, n. 7, p. 495-500, 2009.

GUZMÁN, M. Enseñanza de las ciencias y la matemática. Revista Iberoamericana de Educación, Madrid, v. 43, p. 19-58, 2007.

HABERMAS, J. Teorías de la verdad. In: NICOLÁS, J. A.; M. J. FRÁPOLI, M. J. (Ed.), Teorías de la verdad en el siglo XX. Madrid: Technos, 1997, p. 543-596.

INGVARSON, L.; MEIERS, M.; BEAVIS, A. Factors affecting the impact of professional development programs on teachers' knowledge, practice, student outcomes e efficacy. Education Policy Analysis Archives, Arizona, v. 13, n. 10, p. 1-26, 2005. 
LEE, S. Encyclopedia of school psychology. Thousand Oaks: Sage, 2005.

LINCOLN, Y. S. y GUBA, E. G. Naturalistic inquiry. Beverly Hills: Sage, 1985.

MORALES-LÓPEZ, Y. Costa Rica: The Preparation of Mathematics Teachers. In: RUIZ, Á. (Ed.). Mathematics Teacher Preparation in Central America and the Caribbean. Springer, Cham, 2017, p. 39-56.

MORALES-LÓPEZ, Y.; FONT, V. Valoración realizada por una profesora de la idoneidad de su clase de matemáticas. Educação e Pesquisa, São Paulo, v. 45, e189468, p. 1-10, 2019.

MOREN, E. B. Da S.; DOS SANTOS, Á. R. Uma reflexão sobre ações de formação de professores no Brasil. Revista Iberoamericana de Educación, Madrid, v. 55, n. 1, p. 11, 2011.

NCTM. Principles and Standards for School Mathematics. Reston: VA and National Council of Teachers of Mathematics. 2000.

RAMOS, A. B. Objetos personales, matemáticos y didácticos, del profesorado y cambios institucionales. El caso de la contextualización de las funciones en una Facultad de Ciencias Económicas y Sociales. 2006. Tesis (Doctorado en Didàctica de les Ciències Experimentals i la Matemàtica) - Departamento de Didáctica de la Matemática, Universitat de Barcelona, Barcelona, 2006.

REIS, S. R. Matemática Financeira na perspectiva da Educação Matemática Crítica. 2013. $113 \mathrm{f}$. Dissertação (mestrado) - Mestrado Profissional em Matemática em Rede Nacional - PROFMAT, Centro de Ciências Exatas, Universidade Federal de Santa Maria, Santa Maria, 2013.

RIVAS, M. Análisis epistémico y cognitivo de tareas de proporcionalidad en la formación de profesores de educación primaria. 2013. Tesis (Doctorado en Didáctica de la Matemática) Departamento de Didáctica de la Matemática, Universidad de Granada, Granada, 2013.

ROESKEN, B. Hidden Dimensions in the Professional Development of Mathematics Teachers. Inservice Education for a with teachers. Rotterdam: Sense Publishers, 2011.

RONDERO, C.; FONT, V. Articulación de la complejidad matemática de la media aritmética. Enseñanza de las Ciencias, Barcelona, v. 33, n. 2, p. 29-49, 2015.

SECKEL, M. J. Competencia en análisis didáctico en la formación inicial de profesores de educación básica con mención en matemática. 2016. Tesis (Doctorado en Didàctica de les Ciències Experimentals i la Matemàtica) - Departamento de Didàctica de les Ciències Experimentals i la Matemàtica, Universitat de Barcelona, Barcelona, 2016.

Submetido em 20 de Maio de 2019. Aprovado em 09 de Setembro de 2019. 